\title{
Differences between ketamine's short-term and long-term effects on brain circuitry in depression
}

\author{
Natalia Gass ${ }^{1}$, Robert Becker [1', Jonathan Reinwald ${ }^{1,2}$, Alejandro Cosa-Linan³, Markus Sack', Wolfgang Weber-Fahr ${ }^{1}$, \\ Barbara Vollmayr ${ }^{2,4}$ and Alexander Sartorius ${ }^{1,2}$
}

\begin{abstract}
Ketamine acts as a rapid clinical antidepressant at 25 min after injection with effects sustained for 7 days. As dissociative effects emerging acutely after injection are not entirely discernible from therapeutic action, we aimed to dissect the differences between short-term and long-term response to ketamine to elucidate potential imaging biomarkers of ketamine's antidepressant effect. We used a genetical model of depression, in which we bred depressed negative cognitive state $(\mathrm{NC})$ and non-depressed positive cognitive state $(\mathrm{PC})$ rat strains. Four parallel rat groups underwent stress-escape testing and a week later received either S-ketamine (12 NC, 13 PC) or saline (12 NC, 12 PC). We acquired resting-state functional magnetic resonance imaging time series before injection and at $30 \mathrm{~min}$ and $48 \mathrm{~h}$ after injection. Graph analysis was used to calculate brain network properties. We identified ketamine's distinct action over time in a qualitative manner. The rapid response entailed robust and strain-independent topological modifications in cognitive, sensory, emotion, and reward-related circuitry, including regions that exhibited correlation of connectivity metrics with depressive behavior, and which could explain ketamine's dissociative and antidepressant properties. At $48 \mathrm{~h}$ ketamine had mainly strain-specific action normalizing habenula, midline thalamus, and hippocampal connectivity measures in depressed rats. As these nodes mediate cognitive flexibility impaired in depression, action within this circuitry presumably reflects ketamine's procognitive effects induced only in depressed patients. This finding is especially valid, as our model represents cognitive aspects of depression. These empirically defined circuits explain ketamine's distinct action over time and might serve as translational imaging correlates of antidepressant response in preclinical testing.
\end{abstract}

\section{Introduction}

Major depressive disorder (MDD) is a globally prevalent psychiatric illness with cognitive, affective, and somatic symptoms, including negative emotional states, cognitive impairments, anxiety, anergia, and anhedonia ${ }^{1}$. These symptoms correlate with the aberrant connectivity in

\footnotetext{
Correspondence: Natalia Gass (natalia.gass@zi-mannheim.de)

${ }^{1}$ Research Group Translational Imaging, Department of Neuroimaging, Central Institute of Mental Health, Medical Faculty Mannheim, Heidelberg University, Mannheim, Germany

${ }^{2}$ Department of Psychiatry and Psychotherapy, Central Institute of Mental Health, Medical Faculty Mannheim, Heidelberg University, Mannheim Germany

Full list of author information is available at the end of the article.
}

brain circuits, suggesting it to represent a mechanistic background of depression ${ }^{2,3}$, and implying that MDD is a brain circuit disorder ${ }^{3}$. Recent evidence indicates that ketamine, an NMDA receptor antagonist with rapid antidepressant effects sustained for 7 days $^{4}$, has high potential to normalize disrupted network connectivity, proposing it as the mechanism of action ${ }^{5}$. However, ketamine induces a complex response unfolding distinct influences over time, producing antidepressant effects as fast as $30 \mathrm{~min}$ after administration at $0.5 \mathrm{mg} / \mathrm{kg}$ in humans ${ }^{6}$ and at a comparable dosage of $10 \mathrm{mg} / \mathrm{kg}$ in rat models $^{7,8}$. Yet at the same time, ketamine induces psychotomimetic and dissociative side effects for the first

\section{(c) The Author(s) 2019}

\footnotetext{
(c) Open Access This article is licensed under a Creative Commons Attribution 4.0 International License, which permits use, sharing, adaptation, distribution and reproduction in any medium or format, as long as you give appropriate credit to the original author(s) and the source, provide a link to the Creative Commons license, and indicate if changes were made. The images or other third party material in this article are included in the article's Creative Commons license, unless indicated otherwise in a credit line to the material. If material is not included in the article's Creative Commons license and your intended use is not permitted by statutory regulation or exceeds the permitted use, you will need to obtain permission directly from the copyright holder. To view a copy of this license, visit http://creativecommons.org/licenses/by/4.0/.
} 
$2 h^{9}$. Interestingly, a recent study demonstrated that ketamine's dissociative effects are not entirely discernible from therapeutic action and might even predict a more robust and sustained antidepressant efficacy ${ }^{10}$. This dual action might result from affecting the same multifunctional regions, which are involved in both effects. Facing ketamine's complex action over time, it is important to disentangle the mechanistic network alterations underlying ketamine's activity at different time points.

So far, none of the neuroimaging studies on ketamine directly compared its effects across two time points. Moreover, they were conducted on healthy subjects ${ }^{11-13}$, although ketamine's effects in depressed brain may differ. Those few recent studies that investigated ketamine effects in depressed patients used seed or global connectivity analyses ${ }^{5,14}$. However, since any brain function depends on complex network organization, a quantitative analysis of the brain network architecture using more sophisticated graph theory is highly advantageous, as it dissects network topology and identifies aberrant reconfigurations in functional brain networks.

To address these unresolved issues, we used graph analysis to dissect the relevant circuits for short-term and long-term effects of ketamine in a well-established genetical rat model of depression built upon learned helplessness paradigm in which rats are bred based on their susceptibility to develop stress-escape behavior in operant boxes ${ }^{15}$. The rat strain failing to escape when exposed to electrical footshocks (negative cognitive (NC) state strain) reflects the cognitive dysfunction characteristic for depressive behavior ${ }^{16}$, models treatment-resistant depression ${ }^{15}$ and posttraumatic stress disorder (PTSD) ${ }^{17}$. The term "NC state strain" reflects the pessimistic response bias in cognitive tasks observed in this strain ${ }^{16,18}$. The positive cognitive (PC) state strain displaying escape behavior represents nondepressed phenotype ${ }^{15}$. The model has a high face, construct, and predictive validity ${ }^{15}$. One of its major advantages is that it represents cognitive aspects of depression, in which events are considered negative and uncontrollable, resulting in feelings of anxiety and helplessness. Animal models have the crucial advantage of providing homogenous groups in contrast to human studies, which usually recruit medicated patients with complex and greatly heterogeneous symptoms often influenced by comorbidities with other psychiatric disorders. In addition, animal models are used in preclinical studies to support drug assessment and approvals.

First, we investigated behavioral deficits and network topological alterations in depressed rats to characterize animal model before ketamine administration. Then, we acquired functional magnetic resonance imaging (fMRI) scans at $30 \mathrm{~min}$ and $48 \mathrm{~h}$ post-ketamine and calculated network properties to distinguish ketamine's short-term and long-term impact on brain circuitry.

\section{Methods and materials \\ Experimental design}

Four parallel groups of $\mathrm{NC}$ and PC male rats $(n=49$; 82-83rd genetical generations; Sprague-Dawley; 8 weeks old; 282-414 g) underwent an escape test and 1 week later received a subcutaneous injection of either S-ketamine at $10 \mathrm{mg} / \mathrm{kg}$ (Ketanest, Pfizer Pharma GmBH, Berlin, Germany) (12 NC, $13 \mathrm{PC}$ rats) or saline (12 NC, $12 \mathrm{PC}$ rats), both at volume $2 \mathrm{ml} / \mathrm{kg}$ (Fig. 1). We selected $10 \mathrm{mg} / \mathrm{kg}$ dose based on previously reported antidepressant-like effects in rats ${ }^{19,20}$. This dose also results in a similar range of peak concentrations as $0.5 \mathrm{mg} / \mathrm{kg}$ dose in human studies ${ }^{21}$. We used S-ketamine, since so far it is the only clinically tested enantiomer that was shown to exert rapid antidepressant effects in patients ${ }^{22}$. R-ketamine effects were tested only in rodents $^{23}$, and it remains to be demonstrated whether it has similar antidepressant properties, as S-ketamine in clinical population. Considering the similarity in the pharmacokinetic-pharmacodynamic properties of ketamine in rats and humans ${ }^{24,25}$, and in the timing of its antidepressant action $^{7,8}$, we selected $30 \mathrm{~min}$ and $48 \mathrm{~h}$ for MRI measurements.

Due to the exploratory nature of this study, no formal power or sample size estimation was performed, but the group sizes ( $n=12-13$ per group) are toward the high end of the range typically used in animal fMRI experiments.

The rats were housed in plastic cages, two rats per cage, at constant temperature of $22^{\circ} \mathrm{C}$ and 12-h light-dark cycle (lights on at 07:00 am). Food and water were available ad libitum. The rats were killed at the end of the experiments. We conducted experiments according to the regulations covering animal experimentation within the European Union (European Communities Council Directive 86/609/EEC) and within the German Animal Welfare Act, and were approved by the German animal welfare authorities (Regierungspräsidium Karlsruhe).

\section{Test for escape behavior}

Behavioral experiments were performed, as before ${ }^{26}$, and consisted of an escape paradigm, in which animals could avoid electrical footshocks by pressing lever in operant conditioning chambers (for details see Supplement).

Using nonparametric Kruskal-Wallis test due to nonnormal distribution of the data, we calculated difference between $\mathrm{NC}$ and $\mathrm{PC}$ groups for the following parameters: sum of latencies for trials 3-10, number of failures to terminate shock (failure pattern), and number of trials not stopped within the first $20 \mathrm{~s}$ (deficit pattern) ${ }^{27}$ (significance level at $p<0.05)$. We confirmed previously observed escape deficits in NC rats (Fig. 2, see details in Supplement). 


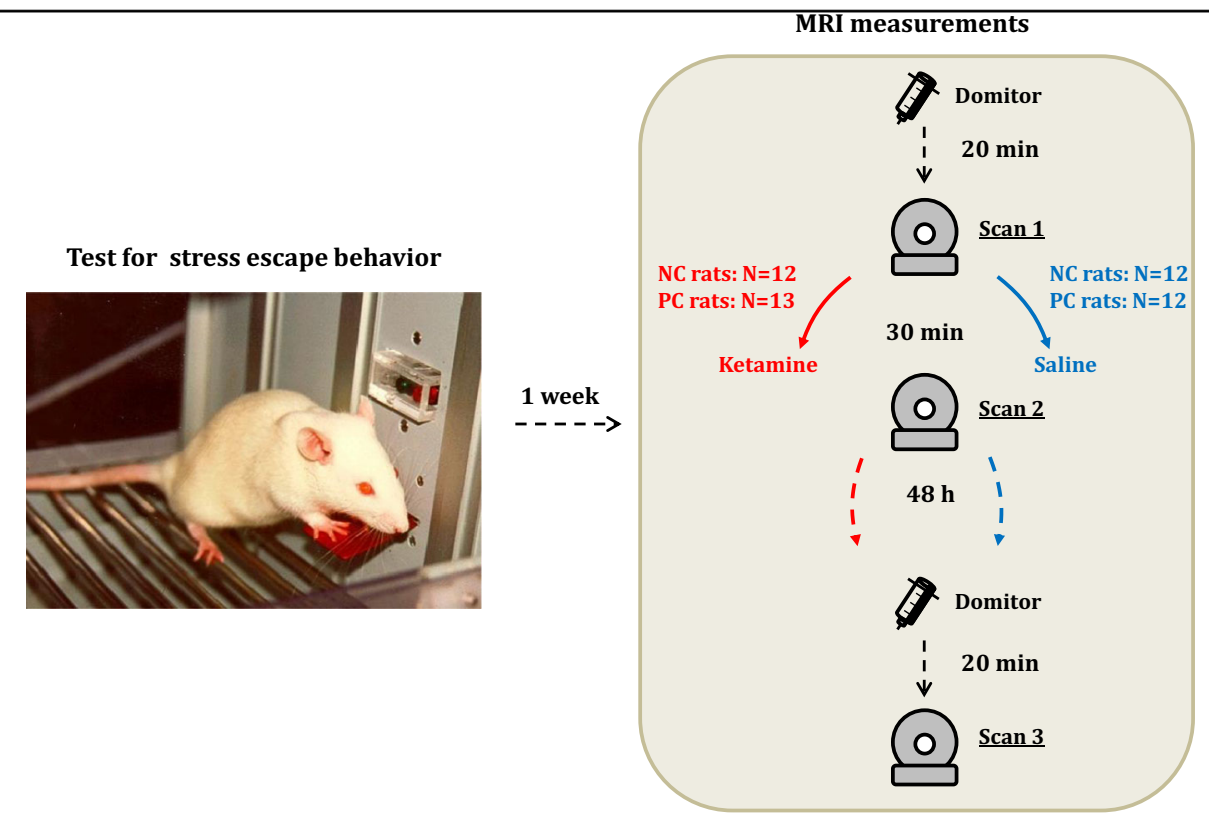

Fig. 1 A scheme of the experimental timeline. The fMRI sessions followed test for escape behavior one week later. They included the baseline preinjection fMRI measurement (scan 1) acquired 20 min after the start of domitor continuous infusion, i.e., after stabilization of respiratory and cardiac rhythms, and the second fMRI scan at 30 min a"fter ketamine/saline injection (scan 2). After $48 \mathrm{~h}$ we repeated the measurement (scan 3). NC negative cognitive state strain, PC - positive cognitive state strain. The image of the rat in operant box is reproduced from "Neurobiologie depressiver Störungen" by Psychiatrie und Psychotherapie Up2date 2010, with permission from Georg Thieme Verlag.

\section{MRI acquisition and preprocessing}

The resting-state fMRI (rs-fMRI) experiments were carried out at 9.4 T MRI scanner (Bruker BioSpec, Ettlingen, Germany) under anesthesia, as previously done $^{26}$. The current data represent a newly acquired MRI dataset, not described in any of our previous stu$\operatorname{dies}^{26,28,29}$. The anesthetic regime was identical to the one described previously ${ }^{26}$. The rats were initially anesthetized with $4 \%$ isoflurane (Baxter Deutschland $\mathrm{GmbH}$, Unterschleissheim, Germany) in a mixture of $\mathrm{N}_{2}(70 \%)$ and $\mathrm{O}_{2}$ (30\%); after positioning in the scanner isoflurane level was reduced to $2.5 \%$ and medetomidine (Domitor, JanssenCilag, Neuss) was first injected as a bolus $(0.5 \mathrm{ml}, 0.07 \mathrm{mg} /$ $\mathrm{kg}$, s.c.) and then after a $10 \mathrm{~min}$ period of a slow discontinuation of isoflurane administration (reduction by $0.5 \%$ every $2 \mathrm{~min}$ before switching off) - as a continuous infusion $(0.28 \mathrm{mg} / \mathrm{kg} / \mathrm{h})$. The sedation depth was monitored via recording the physiological (respiratory and cardiac) parameters throughout the experiment. Breathing and cardiac signals were recorded (10-ms resolution) using the signal breakout module (Small Animal Instruments Inc., NY, USA) and the four-channel recorder (Velleman ${ }^{\oplus}$ N.V., Gavere, Belgium). The physiological parameters stabilized at $15 \mathrm{~min}$ after the start of a continuous medetomidine infusion and stayed stable during the whole experiment.

The MRI acquisition protocol on the day of ketamine/ saline injection included two rs-fMRI time series (pre- injection and post-injection at $30 \mathrm{~min}$ ), FieldMap (before each rs-fMRI for correction of geometric distortions) and structural image (see Supplement for description) (Fig. 1). At $48 \mathrm{~h}$ post-injection the third rs-fMRI time series were acquired along with the FieldMap and structural image. The rs-fMRI T2*-weighted echo-planar imaging-free induction decay (EPI-FID) sequence was acquired using the following parameters: repetition time/echo time (TR/ TE) $1500 / 17.5 \mathrm{~ms}$, flip angle $60^{\circ}$, field of view $35 \times 35 \mathrm{~mm}^{2}$, voxel dimension $0.365 \mathrm{~mm}, 30$ coronal slices (ascending slice order), slice thickness $0.5 \mathrm{~mm}, 340$ acquisitions over $8.5 \mathrm{~min}$, and 8 dummy scans. A 3D double gradient echo FieldMap sequence was obtained using the following parameters: $\mathrm{TR}=20 \mathrm{~ms}$, short $\mathrm{TE}=1.7 \mathrm{~ms}$, and long $\mathrm{TE}$ $=5.7 \mathrm{~ms}$. Structural brain image was acquired using a T2weighted rapid acquisition with refocused echoes (RARE) sequence with the following parameters: RARE factor 16, TR/TE $1200 / 50 \mathrm{~ms}$, flip angle $180^{\circ}$, the voxel dimension $0.15 \mathrm{~mm}$, and acquisition time $23 \mathrm{~min}$.

To compose the daily schedules of fMRI measurements, we used randomization according to the group (PC/NC), treatment (ketamine/saline), and time of the day. During the experiment, the investigator was blinded to the group assignment.

Image preprocessing was performed similarly to our previous studies ${ }^{29,30}$ and included the following steps: (1) correction of each EPI time series for magnetic field (B0) inhomogeneities and movement using "realign and 


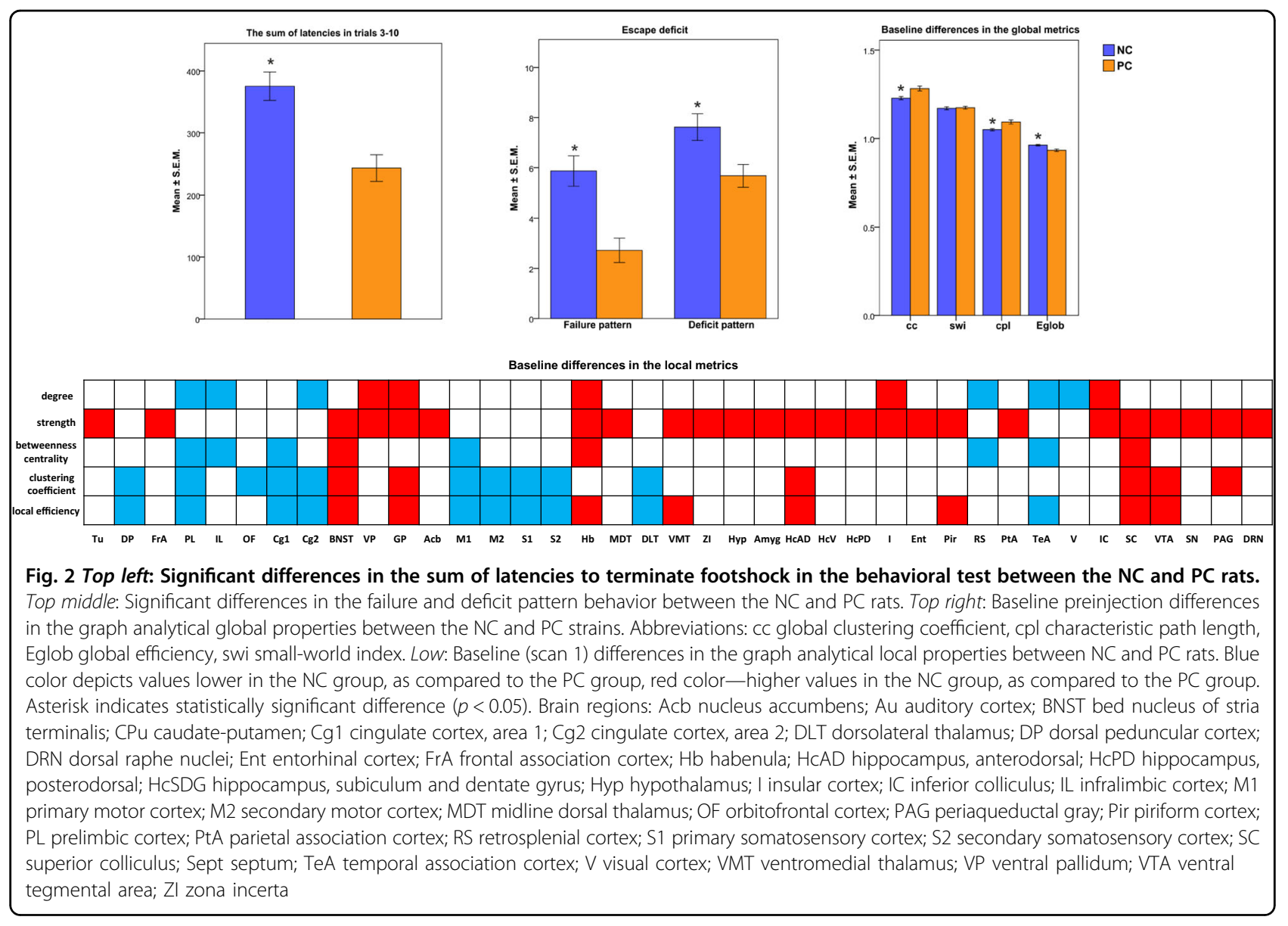

unwarp" SPM function (SPM8: http://www.fil.ion.ucl.ac. $\mathrm{uk} / \mathrm{spm} / \mathrm{software} / \mathrm{spm} 8$ ); (2) regressing out the estimated movement parameter vectors from each voxel (FSL, version 4.1. http://www.fmrib.ox.ac.uk/fsl); (3) filtering out respiratory and cardiac signals using Aztec software ${ }^{31}$; (4) slice-timing correction (SPM8); (5) spatial normalization to a rat brain template in Paxinos space $^{32}$ (SPM8); (6) filtering out the cerebrospinal fluid signal from the normalized images (FSL); (7) frame-wise displacement (FD) and scrubbing to capture the remaining motion-related $\operatorname{artifacts}^{33}$, similarly to our previous study ${ }^{30}$, where we used an ellipsoid-like model instead of a spherical one, considering the non-spherical shape of rat brains compared to humans, and scrubbed all data frames with a FD $>0.03 \mathrm{~mm}$ and interpolated the missing frames using a linear interpolation; (8) the global signal regression; and (9) the band-pass filtering $(0.01-0.1 \mathrm{~Hz})$ (Analysis of Functional NeuroImages version 2$)^{34}$. We note that due to a minimal amount of movement in rats, scrubbing had to be performed only in $\sim 25 \%$ of datasets with removal of only 2-4 volumes in most of the animals. The effects of the preprocessing steps are presented in Supplementary Figs. S1 and S2.

\section{Graph theoretical analysis Graph generation}

Graphs consisted of Pearson correlation coefficients between time courses of 43 bilateral regions covering the whole brain ${ }^{26}$ and defined by Schwarz atlas ${ }^{32}$ (Fig. S3, Table S1). Edge weights of each subject and measurement were normalized, i.e. divided by their maximum correlation value within correlation matrices. The matrices were analyzed in Matlab using version 2015-01-25 of the freely available Brain Connectivity Toolbox ${ }^{35}$.

\section{Integration of network metrics over a range of sparsity thresholds}

Fully-connected weighted graphs were transformed into equi-sparse networks by retaining a fixed percentage of edges. To determine systematic effects on topological organization that would not depend on the choice of a single arbitrary threshold, we selected a range of thresholds. The lower threshold of $25 \%$ corresponded to the value at which networks for each subject and measurement were fully connected, meaning there were no infinite path lengths for any node, as was previously done ${ }^{36}$. At the upper threshold of $45 \%$ no negatively weighted edges were 
present, as previously described ${ }^{26,29}$. After thresholding the networks and calculating graph metrics for each of them, the area under the curve (AUC) was computed for each network metric. The groups were compared based on AUC parameters. The AUC method is sensitive to topological alterations in psychiatric disorders, and thus has been extensively used in brain network studies ${ }^{2,37}$.

\section{Global and local metrics}

For each network and sparsity level, we calculated five global metrics-characteristic path length, global clustering coefficient, small-world index, global efficiency, and local efficiency, and five local metrics-degree, strength, betweenness centrality, clustering coefficient, and local efficiency (see definitions in Supplement).

\section{Correlation of behavior with network properties}

We defined which regions differed in their baseline network properties between the $\mathrm{NC}$ and PC groups using permutation tests $(10,000$ permutations, $n=24 \mathrm{NC}$ rats, $n=25$ PC rats, $p<0.05$ ) (Fig. 2) and then used Spearman tests to correlate those values that differed between the groups with individual behavioral parameters in the NC rats $(p<0.05)$.

\section{Statistical analysis}

To control for possible differences in overall connectivity strength, for each network a resampling algorithm created reference random networks with preserved degree and strength distributions (100 random networks $)^{38}$. To normalize for individual baseline differences, we calculated differences of network properties, subtracting pre-injection values from post-injection (Fig. 1):

$\Delta 1=\operatorname{scan} 2-\operatorname{scan} 1$

$\Delta 2=\operatorname{scan} 3-$ scan 1

For each delta value, we calculated two-way ANOVA with "group" (NC/PC) and "treatment" (ketamine/saline), as factors ( $p<0.05$; false discovery rate correction $q<0.05$ for the number of regions). We chose to analyse the second and the third time points separately as deltas (difference with the first time point) and in a rather qualitative fashion, because the differences to the first time point also include time effects due to prolonged anesthezia which would be difficult to interpret in a repeated measurements ANOVA with three factors.

\section{Results}

The NC rats express a shift toward network randomization and disrupted connectivity within cognitive, anxiogenic, and reward-related circuitries

In the baseline measurement (scan 1) NC rats, compared to $\mathrm{PC}$ rats, exhibited significantly lower global clustering coefficient $(1.23 \pm 0.01$ versus $1.28 \pm 0.01)$ and characteristic path length $(1.05 \pm 0.01$ versus $1.09 \pm 0.01)$, and higher global efficiency $(0.96 \pm 0.00$ versus $0.93 \pm 0.01)$ (Fig. 2), which represent a shift toward random organization.

The prefrontal and somato-motor regions in NC rats displayed lower degree, betweenness centrality and/or clustering, whereas the anxiogenic bed nucleus of stria terminalis and amygdala exhibited higher connectivity measures (Fig. 2, Table S2). Subcortical regions involved in working memory and cognitive flexibility, such as anterodorsal hippocampus, midline thalamus, and habenula, as well as reward-processing areas-ventral pallidum, nucleus accumbens, and ventral tegmental area-manifested increased strength in depressed rats (Fig. 2, Table S2).

Escape deficit correlates with the connectivity metrics of cognitive, motor, anxiogenic, and reward-related nodes

Clustering coefficient of the anterodorsal hippocampus negatively correlated with failure $(r=-0.43 ; p=0.04)$ and deficit pattern $(r=-0.43, p=0.04)$. Somato-motor nodal properties inversely correlated with depressive behavior (Table S3). Amygdala, a key anxiogenic node, manifested a positive correlation of its strength with failure pattern $(r=0.49, p=0.02)$ and sum of latencies $(r$ $=0.45, p=0.03$ ). Reward-related ventral pallidum and ventral tegmental area exhibited positive correlation between their strength and efficiency, and failure pattern ( $r=0.44, p=0.03 ; r=0.45, p=0.03$, respectively).

\section{Ketamine had a robust general effect on local metrics in both strains}

Similarly to our previous study on healthy rats ${ }^{29}$, in both strains ketamine enhanced strength/clustering in the prefrontal regions, such as prelimbic (strength: treatment, $F_{1,45}=5.97, p=0.02$; clustering: treatment, $F_{1,45}=44.96$, $p<0.001$ ), cingulate (strength: treatment, $F_{1,45}=7.49$, $p=0.01$; clustering: treatment, $F_{1,45}=46.47, p<0.001$ ), orbitofrontal (clustering: treatment, $F_{1,45}=28.08$, $p<0.001$ ), and secondary motor cortices (strength: treatment, $F_{1,45}=6.91, p=0.01$ ) (Figs. 3 and 4; Table S4). An opposite change occurred in anterodorsal (strength: treatment, $F_{1,45}=10.08, p<0.01$; clustering: treatment, $\left.F_{1,45}=22.58, p<0.001\right)$ and posterodorsal hippocampus (strength: treatment, $F_{1,45}=8.00, p=0.01$; clustering: treatment, $F_{1,45}=15.35, p<0.01$ ) (Figs. 3 and 4; Table S4). Also, ketamine reduced strength in amygdala (treatment, $\left.F_{1,45}=5.61, p=0.02\right)$, and strength/clustering in nucleus accumbens (clustering: treatment, $F_{1,45}=5.96, p=0.02$ ), ventral pallidum (strength: treatment, $F_{1,45}=5.87, p=$ 0.02 ), and ventral tegmental area (strength: treatment, $F_{1,45}=8.61, p=0.01$ ) (Figs. 3 and 4 ).

\section{Ketamine had a short-lasting strain-unspecific effect on global metrics}

The change in global topological properties in response to ketamine was short-lasting (Fig. 5, Table S5), 


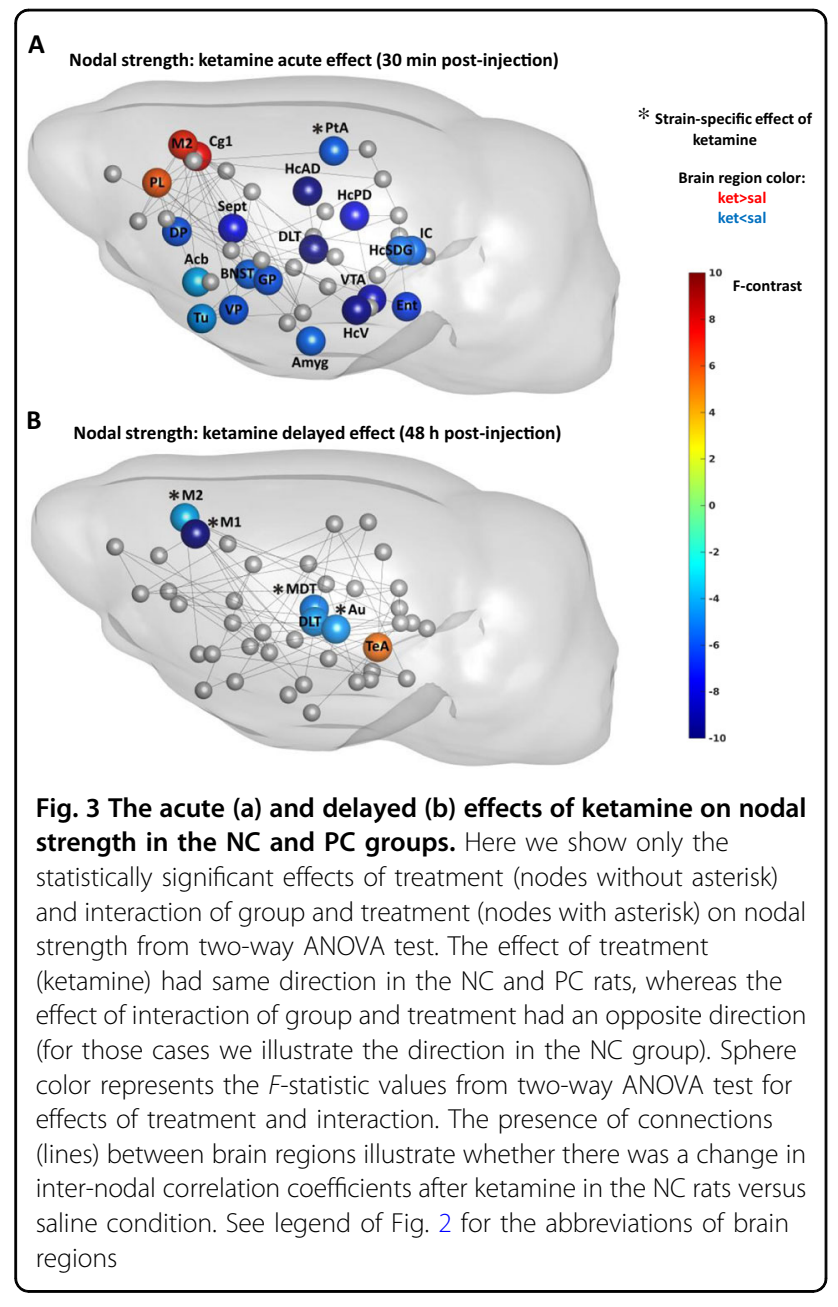

with no effects sustained $48 \mathrm{~h}$ later. The common response in both strains, as compared to respective control groups, was an increase in clustering coefficient (treatment, $F_{1,45}=16.84, p<0.001$ ) and path length (treatment, $F_{1,45}=36.48, p<0.001$ ), indicating increased network segregation and decreased integration, and a decrease in small-world index (treatment, $F_{1,45}=6.41$, $p=0.01$ ) and global efficiency (treatment, $F_{1,45}=45.20$, $p<0.001)$.

\section{Ketamine had few strain-specific short-term effects}

Depressed rats, contrary to PC rats, exhibited reduction of strength/clustering in nodes involved in cognitive processing, such as parietal association cortex (strength: interaction, $F_{1,45}=5.83, p=0.02$ ) and subiculum and dentate gyrus regions of the hippocampus (clustering: interaction, $F_{1,45}=5.28, p=0.03$ ) (Figs. 3 and 4). Also, ketamine raised clustering of bed nucleus of stria terminalis (interaction, $F_{1,45}=5.94, p=0.02$ ) in $\mathrm{NC}$ rats. The only node that showed the same direction of change in both groups, albeit in different extent, was the auditory

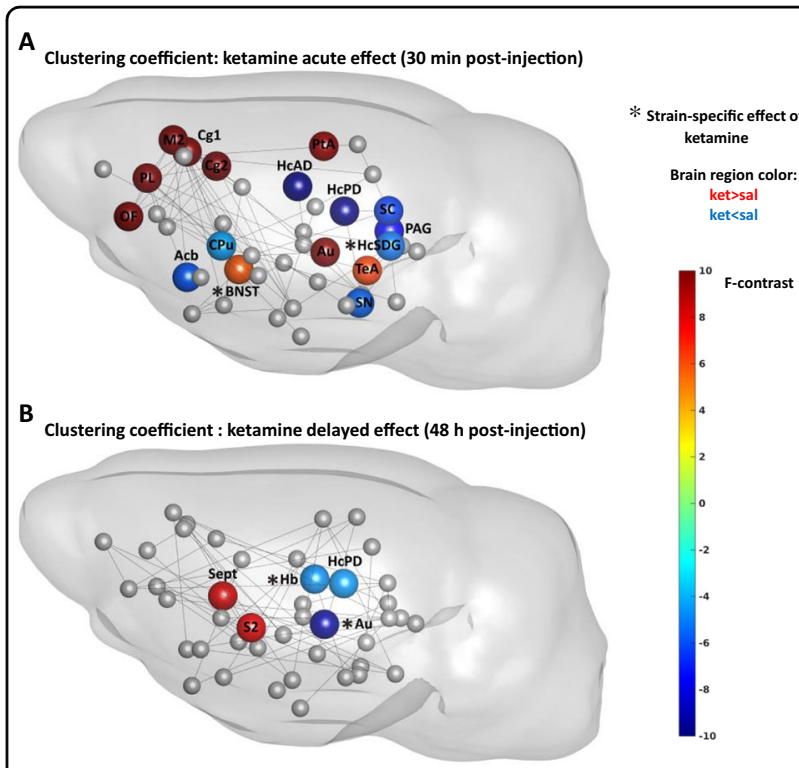

Fig. 4 The acute (a) and delayed (b) effects of ketamine on nodal clustering coefficient in the NC and PC groups. See legend of Fig. 3 for the detailed description

cortex, exhibiting increased efficiency (interaction, $F_{1,45}=$ $5.97, p=0.02$ ).

Ketamine exhibited mainly strain-specific long-term effects

While most of ketamine's acute effects were strainindependent, the delayed effects were mainly strainspecific (Figs. 3-5, Table S6) and expressed opposite directions between strains.

The strain-specific response included nodes that did not appear at acute stage, such as habenula displaying reduced clustering (interaction, $F_{1,45}=4.71, p=0.04$ ) and efficiency (interaction, $F_{1,45}=4.41, p=0.04$ ), and midline thalamus exhibiting reduced degree (interaction, $F_{1,45}=$ $4.27, p=0.04)$ and strength in depressed rats (interaction, $\left.F_{1,45}=5.12, p=0.03\right)$.

In addition, we observed diminished connectivity measures in the auditory (strength: interaction, $F_{1,45}=4.66$, $p=0.04$; betweenness centrality: interaction, $F_{1,45}=6.17$, $p=0.02$; clustering: interaction, $F_{1,45}=9.45, p<0.01$; efficiency: interaction, $\left.F_{1,45}=11.23, p<0.01\right)$ and primary motor cortices (degree: interaction, $F_{1,45}=11.42, p<0.01$; strength: interaction, $F_{1,45}=10.79, p<0.01$ ).

\section{Discussion}

Regions that manifested the strongest strain baseline differences and showed correlation with depressive behavior, covered areas belonging to top $10 \%$ with the most abnormal connectivity features shared by four major depression types $^{3}$. Ketamine effects on global topological organization were short-lasting and in opposite direction to patterns in 


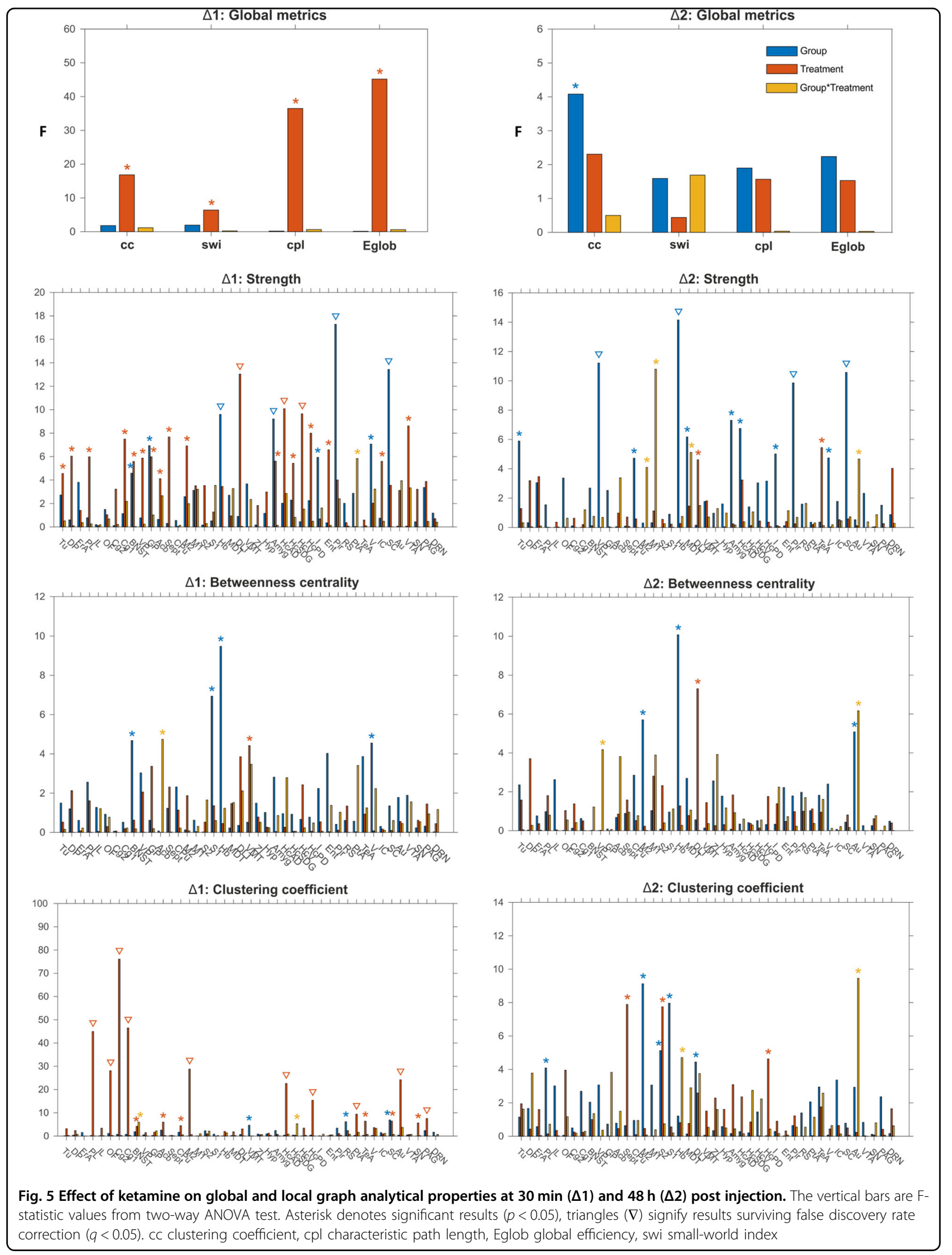


depressed brain. Ketamine's local action encompassed rapid strain-independent robust modification within the cognitive, sensory, subcortical emotion, and reward-related circuitry, including regions that exhibited correlation of their connectivity metrics with depressive behavior, and delayed strain-specific long-term normalization of connectivity measures for nodes mediating cognitive flexibility.

\section{Shift toward brain network randomization in depressed rats}

Similarly to depressed patients, NC rats exhibited decreased global clustering ${ }^{39-41}$ and path length ${ }^{2,40,42}$, and enhanced global efficiency ${ }^{2,40}$, which signify decreased segregation and a shift toward brain network randomization. Two clinical studies report diminished global efficiency and increased characteristic path length ${ }^{39,43}$. However, the discrepancy of graph analytical findings in different studies is not surprising, given symptomatic heterogeneity of depression and the predominantly medicated state of patients, except two studies on drugnaïve subjects and patients after a medication washout $^{2,41}$, both of which validate our results.

\section{Strain baseline differences in network local topology}

$\mathrm{NC}$ rats manifested largely reduced prefrontal connectivity metrics, which is one of the most replicated findings in unmedicated depressed and PTSD patients ${ }^{44-46}$. This hypoconnectivity could result in blunted prefrontal top-down control over limbic system leading to enhanced amygdala activation and negative bias ${ }^{47}$. Consistently with this suggestion, we detected increased amygdala strength in the NC group, which positively correlated with depressive behavior. Furthermore, these changes might also contribute to enhanced freezing resistant to extinction in $\mathrm{NC}$ rats ${ }^{17}$, resembling persistent fear-related behavior in nonthreatening situations in MDD and $\mathrm{PTSD}^{48}$, as consolidation of fear extinction occurs within the infralimbicamygdala-midthalamic circuit $^{49}$. Accordingly, NC rats exhibited increased strength for all regions of this circuit.

Another finding replicating human studies was increased connectivity measures of hippocampus and negative correlation of its metrics with depression severity ${ }^{2,50}$. Considered together with increased amygdala strength, it might reflect negative cognitive bias in which these regions facilitate recall of negative information ${ }^{1}$. On the other hand, finding lever to terminate footshock in escape test requires good navigation abilities and spatial memory, and since hippocampus mediates these functions $^{51}$, our results might likewise mean that hippocampal hypoconnectivity could underlie impaired spatial cognition and memory across trials and consequently lead to worsened performance.

Next, habenula displayed higher degree, strength, centrality, and efficiency in the $\mathrm{NC}$ group, indirectly replicating habenula hyperactivity characteristic for depression ${ }^{52,53}$. Habenula mediates cognitive flexibility based on aversive events or context change ${ }^{54}$. We suggest that aberrant connectivity in habenula would result in impaired ability to switch behaviors adaptively despite negative outcomes. In addition, habenula suppresses motor activity ${ }^{55}$. Consistently, we found reduced somato-motor nodal properties in NC rats, as well as negative correlation of these regions connectivity with depressive behavior. Given that these regions control motor behavior, their diminished communication may directly affect behavioral performance.

Finally, NC rats demonstrated higher connectivity in reward-related regions, encompassing nucleus accumbens, ventral pallidum, and ventral tegmental area. Accumbens' hyperconnectivity along with prefrontal hypoconnectivity might reflect two independent pathways triggering depression ${ }^{56}$. Accumbens' primary output, ventral pallidum, processes both rewarding and aversive stimuli, and drives positive reinforcement ${ }^{57}$. Pallidal neurons exhibit elevated activity in depression ${ }^{58}$. In line with these data, we have detected positive correlation between pallidal strength and failure pattern. Furthermore, pallidal neurons enhance firing activity of habenula and ventral tegmental area ${ }^{57,59}$, both of which also displayed heightened strength and efficiency in NC rats.

\section{Ketamine's acute strain-independent effects}

Notably, the change in global topology in response to ketamine was short-lasting, with no effects sustained at $48 \mathrm{~h}$. This leads to a conclusion that global topological reorganization is not required for ketamine's long-term antidepressant effect, which rather builds upon fine-tuned topological modifications of specific nodes.

Ketamine acutely reduced strength of several regions that exhibited correlation of their metrics with depressive behavior, namely amygdala, anterodorsal hippocampus and ventral pallidum. Although the effect was strainindependent, it was in opposite direction to the baseline scan of NC rats. Modifying topological properties of these regions, ketamine presumably exerts anti-anxious, procognitive, and reinforcing effects. This normalization response did not sustain after $48 \mathrm{~h}$, nevertheless, it might constitute a part of rapid antidepressant action.

Other common modifications included an increase in prefrontal strength and segregation, which we also detected earlier in healthy rats ${ }^{29}$ and is one of the most consistently replicated findings ${ }^{56}$, essential for ketamine treatment response and predicting treatment outcome $e^{5,60}$. It probably reflects ketamine-induced transient prefrontal glutamate surge and might underlie both dissociative and antidepressant effects ${ }^{21}$. Another effect that robustly replicates human studies was reduction of accumbens' connectivity $^{56}$. Reward-related nucleus accumbens and ventral tegmental area reduced their strength in response 
to ketamine, presumably reflecting ketamine's positive reinforcement action.

Finally, motor cortex displayed increased strength, clustering, and efficiency after ketamine in both groups. This could explain a hyperlocomotion phenomenon after low-dose ketamine administration ${ }^{61}$.

Since all of these changes were in opposite direction to the baseline pattern in NC rats, we suggest that, although ketamine acted strain-independently, it was nevertheless capable to rapidly normalize topology within the cognitive, anxiogenic and reward-related regions in depressed rats, which could explain its quick antidepressant efficacy.

\section{Ketamine's acute strain-specific effects}

The parietal association cortex manifested strength change with opposite direction between strains. As this region integrates sensory information ${ }^{62}$, our result might reflect a difference in ketamine's action between depressed and healthy individuals-interestingly, ketamine induces acute inner tension and somatic anxiety only in healthy individuals ${ }^{63}$.

Bed nucleus of stria terminalis and subiculum/dentate gyrus exhibited strain-specific contrary changes in clustering. Stria terminalis, as part of extended amygdala, is directly involved in expression of sustained anxiety ${ }^{64,65}$, therefore, this alteration may explain the feeling of heightened anxiety induced by ketamine specifically in healthy subjects ${ }^{63}$. Also, ketamine has differential effects on memory, transiently impairing it in healthy humans ${ }^{66,67}$, while improving in depressed patients ${ }^{68}$. As hippocampus participates in memory retrieval ${ }^{69}$, we suggest that strain-specific changes in hippocampal clustering could partially underlie this difference.

Finally, the auditory cortex displayed increased efficiency in both strains, differing only in extent and resembling response in healthy rats from our previous study $^{29}$. Ketamine causes illusory auditory experiences ${ }^{70}$, which are generally associated with activation in the auditory cortex ${ }^{71}$. We reason that increased efficiency of the auditory cortex might reflect ketamine-induced auditory perceptual changes.

\section{Strain-specific long-term effects}

Ketamine's strain-specific long-term effects included normalization of connectivity measures for habenula and midline thalamus. Importantly, these two regions along with hippocampus, whose connectivity was also normalized at both time points, mediate cognitive flexibility $^{72,73}$-a skill profoundly impaired in MDD and PTSD $^{73,74}$, when individuals are unable to process contextual information, distinguish contextual cues in safe versus threatening situations and to modulate fear and emotional response in non-threatening environment. Cognitive flexibility critically depends on contextual encoding and memory processing ${ }^{54}$, which is also impaired in MDD and congenital learned helplessness model $^{75}$. CA1-CA3 regions of the hippocampus, covered by the anterodorsal and posterodorsal seeds in our study, create distinct representation pattern for each situation ${ }^{69}$, thus allowing avoidance of context overgeneralization characteristic for depressed state. Midline thalamus is a partner of hippocampus in forming memories ${ }^{51,72}$. Ketamine improves memory in depressed patients, in contrast to healthy subjects; ${ }^{68}$ also, it ameliorates cognitive flexibility deficit in chronically stressed rats at the same dose like in our study ${ }^{76}$. Since we utilized cognitive model of depression, we suggest that ketamine mediates its procognitive effects by normalizing the disrupted wiring within the habenula-midthalamic-hippocampal cognitive circuitry, which might be a key imaging correlate of its long-term effect.Also, our result of reduced connectivity metrics of habenula may correspond to ketamine-induced reduction of habenula metabolism in patients with treatment-resistant depression ${ }^{77}$ and inhibition of habenula burst firing activity in learned helplessness model $^{78}$, both producing antidepressant action.

Ketamine had a strain-specific effect on the auditory cortex, consistently reducing almost all of its connectivity metrics in NC rats and retaining them raised in PC rats. Since in the baseline scan depressed rats did not show differences in the auditory cortex connectivity measures, this action does not appear to constitute a part of ketamine's antidepressant effect, but might rather represent a lasting modification of the auditory system function. Most studies report a ketamine-induced acute reduction of auditory event-related potentials parameters and mismatch negativity amplitude ${ }^{79,80}$, which might underlie changes in auditory perception, however, the duration of this effect remains to be investigated. On the other hand, since anxiety is characterized by hypervigilance and scanning for potentially threatening auditory cues, downregulation of auditory system might be part of calming effect.

\section{Limitations}

We did not test antidepressant effects of ketamine, since it would greatly interfere with the whole experimental setup, when done between fMRI sessions, and at later stage two scanning sessions would affect test results as stress factors. Previous works already revealed ketamine antidepressant action in learned helplessness behavior ${ }^{7,81,82}$. Although it remains to be demonstrated that our findings would predict treatment response, they illustrate the potential of these empirically defined circuits to serve as benchmarks of ketamine's response.

\section{Conclusion}

Using the negative cognition model of depression, we identified ketamine's qualitatively distinct action over 
time. The rapid topological modification within the cognitive, sensory, and subcortical emotion and rewardrelated circuitry could explain both dissociative and antidepressant effects. The delayed strain-specific normalization of disrupted connectivity within the habenulamidthalamic-hippocampal circuitry presumably reflects ketamine's procognitive efficacy observed only in depressed patients. This finding is especially valid, as we used model representing cognitive aspect of depression and might serve as translational imaging correlates of antidepressant response in preclinical testing.

\section{Acknowledgements}

The authors thank Felix Hörner, Helene Schamber, and Claudia Falfan-Melgoza for their superb technical assistance. This work was supported by the grant from the German Research Foundation (Deutsche Forschungsgemeinschaft): DFG GA 2109/2-1 to N.G. and DFG SA 1869/15-1 to N.G. and A.S., and partially: DFG SA 1869/11-2 within the priority program (SPP1629) "Thyroid Trans Act" (A.S. and J.R.) and Bundesministerium für Bildung und Forschung e:Med program grant (FKZ: 01ZX1311A, FKZ: 01ZX1503) (A.C.-L.).

\section{Author details}

'Research Group Translational Imaging, Department of Neuroimaging, Central Institute of Mental Health, Medical Faculty Mannheim, Heidelberg University, Mannheim, Germany. ${ }^{2}$ Department of Psychiatry and Psychotherapy, Central Institute of Mental Health, Medical Faculty Mannheim, Heidelberg University, Mannheim, Germany. ${ }^{3}$ Research Group In Silico Pharmacology, Central Institute of Mental Health, Medical Faculty Mannheim, Heidelberg University, Mannheim, Germany. ${ }^{4}$ Research Group Animal Models in Psychiatry, Department of Psychiatry and Psychotherapy, Central Institute of Mental Health, Medical Faculty Mannheim, Heidelberg University, Mannheim, Germany

\section{Conflict of interest}

The authors declare that they have no conflict of interest.

\section{Publisher's note}

Springer Nature remains neutral with regard to jurisdictional claims in published maps and institutional affiliations.

Supplementary Information accompanies this paper at (https://doi.org/ 10.1038/s41398-019-0506-6).

Received: 5 October 2018 Revised: 13 March 2019 Accepted: 23 March 2019

Published online: 28 June 2019

\section{References}

1. Disner, S. G., Beevers, C. G., Haigh, E. A. \& Beck, A. T. Neural mechanisms of the cognitive model of depression. Nat. Rev. Neurosci. 12, 467-477 (2011).

2. Zhang, J. et al. Disrupted brain connectivity networks in drug-naive, firstepisode major depressive disorder. Biol. Psychiatry 70, 334-342 (2011).

3. Drysdale, A. T. et al. Resting-state connectivity biomarkers define neurophysiological subtypes of depression. Nat. Med. 23, 28-38 (2017).

4. Zarate, C. A. Jr et al. A randomized trial of an N-methyl-D-aspartate antagonist in treatment-resistant major depression. Arch. Gen. Psychiatry 63, 856-864 (2006).

5. Abdallah, C. G. et al. Ketamine treatment and global brain connectivity in major depression. Neuropsychopharmacology 42, 1210-1219 (2017).

6. Liebrenz, M., Stohler, R. \& Borgeat, A. Repeated intravenous ketamine therapy in a patient with treatment-resistant major depression. World J. Biol. Psychiatry 10, 640-643 (2009).
7. Koike, H., lijima, M. \& Chaki, S. Involvement of AMPA receptor in both the rapid and sustained antidepressant-like effects of ketamine in animal models of depression. Behav. Brain. Res. 224, 107-111 (2011).

8. Fukumoto, K. et al. Antidepressant potential of (R)-ketamine in rodent models: comparison with (S)-ketamine. J. Pharmacol. Exp. Ther. 361, 9-16 (2017).

9. Driesen, N. R. et al. Relationship of resting brain hyperconnectivity and schizophrenia-like symptoms produced by the NMDA receptor antagonist ketamine in humans. Mol. Psychiatry 18, 1199-1204 (2013).

10. Luckenbaugh, D. A. et al. Do the dissociative side effects of ketamine mediate its antidepressant effects? J. Affect Disord. 159, 56-61 (2014).

11. Joules, R. et al. Ketamine induces a robust whole-brain connectivity pattern that can be differentially modulated by drugs of different mechanism and clinical profile. Psychopharmacol. (Berl.) 232, 4205-4218 (2015).

12. Scheidegger, M. et al. Ketamine decreases resting state functional network connectivity in healthy subjects: Implications for antidepressant drug action. PLoS One 7, e44799 (2012).

13. Kraguljac, N. V. et al. Ketamine modulates hippocampal neurochemistry and functional connectivity: A combined magnetic resonance spectroscopy and resting-state fMRI study in healthy volunteers. Mol. Psychiatry 22, 562-569 (2017).

14. Downey, D. et al. Comparing the actions of lanicemine and ketamine in depression: key role of the anterior cingulate. Eur. Neuropsychopharmacol. $\mathbf{2 6}$ 994-1003 (2016).

15. Vollmayr, B. \& Gass, P. Learned helplessness: unique features and translational value of a cognitive depression model. Cell Tissue Res. 354, 171-178 (2013).

16. Enkel, T. et al. Ambiguous-cue interpretation is biased under stress- and depression-like states in rats. Neuropsychopharmacology 35, 1008-1015 (2010).

17. Shumake, J., Barrett, D. \& Gonzalez-Lima, F. Behavioral characteristics of rats predisposed to learned helplessness: reduced reward sensitivity, increased novelty seeking, and persistent fear memories. Behav. Brain. Res. 164, 222-230 (2005).

18. Richter, S. H. et al. A glass full of optimism: enrichment effects on cognitive bias in a rat model of depression. Cogn. Affect. Behav. Neurosci. 12, 527-542 (2012).

19. Li, N. et al. mTOR-dependent synapse formation underlies the rapid antidepressant effects of NMDA antagonists. Science 329, 959-964 (2010).

20. Amat, J. et al. Previous ketamine produces an enduring blockade of neurochemical and behavioral effects of uncontrollable stress. J. Neurosci. 36, 153-161 (2016).

21. Chowdhury, G. M. et al. Transiently increased glutamate cycling in rat PFC is associated with rapid onset of antidepressant-like effects. Mol. Psychiatry 22, 120-126 (2017).

22. Singh, J. B. et al. Intravenous esketamine in adult treatment-resistant depression: a double-blind, double-randomization, placebo-controlled study. Biol. Psychiatry 80, 424-431 (2016).

23. Yang, C. et al. R-ketamine: a rapid-onset and sustained antidepressant without psychotomimetic side effects. Transl. Psychiatry 5, e632 (2015).

24. Edwards, S. R. \& Mather, L. E. Tissue uptake of ketamine and norketamine enantiomers in the rat: Indirect evidence for extrahepatic metabolic inversion. Life. Sci. 69, 2051-2066 (2001).

25. Hoflich, A. et al. Ketamine-dependent neuronal activation in healthy volunteers. Brain. Struct. Funct. 222, 1533-1542 (2017).

26. Gass, N. et al. Brain network reorganization differs in response to stress in rats genetically predisposed to depression and stress-resilient rats. Transl. Psychiatry 6, e970 (2016).

27. Richter, S. H., Sartorius, A., Gass, P. \& Vollmayr, B. A matter of timing: harm reduction in learned helplessness. Behav. Brain. Funct. 10, 41,9081-10-41 (2014).

28. Gass, N. et al. Sub-anesthetic ketamine modulates intrinsic BOLD connectivity within the hippocampal-prefrontal circuit in the rat. Neuropsychopharmacology 39, 895-906 (2014).

29. Gass, N. et al. Antagonism at the NR2B subunit of NMDA receptors induces increased connectivity of the prefrontal and subcortical regions regulating reward behavior. Psychopharmacol. (Berl.) 235, 1055-1068 (2018).

30. Reinwald J. R. et al. Neural mechanisms of early-life social stress as a developmental risk factor for severe psychiatric disorders. Biol. Psychiatry $\mathbf{8 4}$ 116-128 (2018).

31. van Buuren, M. et al. Cardiorespiratory effects on default-mode network activity as measured with fMRl. Hum. Brain. Mapp. 30, 3031-3042 (2009). 
32. Schwarz, A. J. et al. A stereotaxic MRI template set for the rat brain with tissue class distribution maps and co-registered anatomical atlas: application to pharmacological MRI. Neuroimage 32, 538-550 (2006).

33. Power, J. D. et al. Methods to detect, characterize, and remove motion artifact in resting state fMRI. Neuroimage 84, 320-341 (2014).

34. Cox, R. W. AFNl: software for analysis and visualization of functional magnetic resonance neuroimages. Comput. Biomed. Res. 29, 162-173 (1996).

35. Rubinov, M. \& Sporns, O. Complex network measures of brain connectivity: uses and interpretations. Neuroimage 52, 1059-1069 (2010).

36. Bordier, C., Nicolini, C. \& Bifone, A. Graph analysis and modularity of brain functional connectivity networks: searching for the optimal threshold. Front. Neurosci. 11, 441 (2017).

37. Wang, J. et al. Parcellation-dependent small-world brain functional networks: a resting-state fMRI study. Hum. Brain. Mapp. 30, 1511-1523 (2009).

38. Rubinov, M. \& Sporns, O. Weight-conserving characterization of complex functional brain networks. Neuroimage 56, 2068-2079 (2011).

39. Luo, Q. et al. Frequency dependant topological alterations of intrinsic functional connectome in major depressive disorder. Sci. Rep. 5, 9710 (2015).

40. Li, H., Zhou, H., Yang, Y., Wang, H. \& Zhong, N. More randomized and resilient in the topological properties of functional brain networks in patients with major depressive disorder. J. Clin. Neurosci. 44, 274-278 (2017).

41. Gong, L. et al. Disrupted topology of hippocampal connectivity is associated with short-term antidepressant response in major depressive disorder. J. Affect Disord. 225, 539-544 (2018).

42. Hou, Z. et al. Divergent topological architecture of the default mode network as a pretreatment predictor of early antidepressant response in major depressive disorder. Sci. Rep. 6, 39243 (2016).

43. Meng, C. et al. Aberrant topology of striatum's connectivity is associated with the number of episodes in depression. Brain 137, 598-609 (2014).

44. Sripada, R. K. et al. Neural dysregulation in posttraumatic stress disorder: evidence for disrupted equilibrium between salience and default mode brain networks. Psychosom. Med. 74, 904-911 (2012).

45. Sawaya $H$. et al. Resting-state functional connectivity of antero-medial prefrontal cortex sub-regions in major depression and relationship to emotional intelligence. Int. J. Neuropsychopharmacol. 18, (2015).

46. Scheinost, D. et al. Multimodal investigation of network level effects using intrinsic functional connectivity, anatomical covariance, and structure-tofunction correlations in unmedicated major depressive disorder. Neuropsychopharmacology 43, 1119-1127 (2018).

47. Sinha, R., Lacadie, C. M., Constable, R. T. \& Seo, D. Dynamic neural activity during stress signals resilient coping. Proc. Natl Acad. Sci. USA 113, 8837-8842 (2016).

48. Kheirbek, M. A., Klemenhagen, K. C., Sahay, A. \& Hen, R. Neurogenesis and generalization: a new approach to stratify and treat anxiety disorders. Nat. Neurosci. 15, 1613-1620 (2012).

49. Lee, S. et al. Bidirectional modulation of fear extinction by mediodorsal thalamic firing in mice. Nat. Neurosci. 15, 308-314 (2011).

50. Guo, H. et al. Resting-state functional connectivity abnormalities in first-onset unmedicated depression. Neural Regen. Res. 9, 153-163 (2014).

51. Bannerman, D. M. et al. Hippocampal synaptic plasticity, spatial memory and anxiety. Nat. Rev. Neurosci. 15, 181-192 (2014).

52. Sartorius, A. et al. Remission of major depression under deep brain stimulation of the lateral habenula in a therapy-refractory patient. Biol. Psychiatry 67, e9-e11 (2010).

53. Cui, W. et al. Glial dysfunction in the mouse habenula causes depressive-like behaviors and sleep disturbance. J. Neurosci. 34, 16273-16285 (2014).

54. Mizumori, S. J. Y. \& Baker, P. M. The lateral habenula and adaptive behaviors. Trends Neurosci. 40, 481-493 (2017).

55. Hikosaka, O. The habenula: from stress evasion to value-based decisionmaking. Nat. Rev. Neurosci. 11, 503-513 (2010).

56. Abdallah, C. G., Sanacora, G., Duman, R. S. \& Krystal, J. H. The neurobiology of depression, ketamine and rapid-acting antidepressants: Is it glutamate inhibition or activation? Pharmacol. Ther. 190, 148-158 (2018).

57. Tooley, J. et al. Glutamatergic ventral pallidal neurons modulate activity of the habenula-tegmental circuitry and constrain reward seeking. Biol. Psychiatry $\mathbf{8 3}$, 1012-1023 (2018).

58. Knowland, D. et al. Distinct ventral pallidal neural populations mediate separate symptoms of depression. Cell 170, 284-297 (2017).
59. Faget, L. et al. Opponent control of behavioral reinforcement by inhibitory and excitatory projections from the ventral pallidum. Nat. Commun. 9, 849 (2018).

60. Abdallah, C. G. et al. Prefrontal connectivity and glutamate transmission: relevance to depression pathophysiology and ketamine treatment. Biol. Psychiatry Cogn. Neurosci. Neuroimaging 2, 566-574 (2017).

61. Hakami, T. et al. NMDA receptor hypofunction leads to generalized and persistent aberrant gamma oscillations independent of hyperlocomotion and the state of consciousness. PLOS ONE 4, e6755 (2009).

62. Whitlock, J. R., Sutherland, R. J., Witter, M. P., Moser, M. B. \& Moser, E. I. Navigating from hippocampus to parietal cortex. Proc. Natl Acad. Sci. USA 105, 14755-14762 (2008).

63. Nugent, A. C. et al. Ketamine has distinct electrophysiological and behavioral effects in depressed and healthy subjects. Mol. Psychiatry 24, 1040-1052 (2018).

64. Adhikari, A. Distributed circuits underlying anxiety. Front. Behav. Neurosci. 8, 112 (2014).

65. Davis, M., Walker, D. L., Miles, L. \& Grillon, C. Phasic vs sustained fear in rats and humans: Role of the extended amygdala in fear vs anxiety. Neuropsychopharmacology 35, 105-135 (2010).

66. Adler, C. M., Goldberg, T. E., Malhotra, A. K., Pickar, D. \& Breier, A. Effects of ketamine on thought disorder, working memory, and semantic memory in healthy volunteers. Biol. Psychiatry 43, 811-816 (1998).

67. Morgan, C. J. \& Curran, H. V. Acute and chronic effects of ketamine upon human memory: a review. Psychopharmacol. (Berl.) 188, 408-424 (2006).

68. Lee, $Y$. et al. A new perspective on the anti-suicide effects with ketamine treatment: a procognitive effect. J. Clin. Psychopharmacol. 36, 50-56 (2016).

69. Bakker, A., Kirwan, C. B., Miller, M. \& Stark, C. E. Pattern separation in the human hippocampal CA3 and dentate gyrus. Science 319, 1640-1642 (2008).

70. Krystal, J. H. et al. Subanesthetic effects of the noncompetitive NMDA antagonist, ketamine, in humans. psychotomimetic, perceptual, cognitive, and neuroendocrine responses. Arch. Gen. Psychiatry 51, 199-214 (1994).

71. Shergill, S. S. et al. Modality specific neural correlates of auditory and somatic hallucinations. J. Neurol. Neurosurg. Psychiatry 71, 688-690 (2001).

72. Parnaudeau, S., Bolkan, S. S. \& Kellendonk, C. The mediodorsal thalamus: an essential partner of the prefrontal cortex for cognition. Biol. Psychiatry 83, 648-656 (2018).

73. van Rooij, S. J. H. et al. The role of the hippocampus in predicting future posttraumatic stress disorder symptoms in recently traumatized civilians. Biol. Psychiatry 84, 106-115 (2018).

74. Pehrson, A. L. et al. Treatment of cognitive dysfunction in major depressive disorder-a review of the preclinical evidence for efficacy of selective serotonin reuptake inhibitors, serotonin-norepinephrine reuptake inhibitors and the multimodal-acting antidepressant vortioxetine. Eur. J. Pharmacol. 753, 19-31 (2015).

75. Richter, S. H. et al. Where have I been? Where should I go? Spatial working memory on a radial arm maze in a rat model of depression. PLOS ONE $\mathbf{8}$, e62458 (2013).

76. Jett, J. D. et al. Antidepressant-like cognitive and behavioral effects of acute ketamine administration associated with plasticity in the ventral hippocampus to medial prefrontal cortex pathway. Psychopharmacol. (Berl.) 232, 3123-3133 (2015).

77. Carlson, P. J. et al. Neural correlates of rapid antidepressant response to ketamine in treatment-resistant unipolar depression: a preliminary positron emission tomography study. Biol. Psychiatry 73, 1213-1221 (2013).

78. Yang, Y. et al. Ketamine blocks bursting in the lateral habenula to rapidly relieve depression. Nature 554, 317-322 (2018).

79. Rosburg, T. \& Kreitschmann-Andermahr, I. The effects of ketamine on the mismatch negativity (MMN) in humans - a meta-analysis. Clin. Neurophysiol. 127, 1387-1394 (2016).

80. Schwertner, A., Zortea, M., Torres, F. V. \& Caumo, W. Effects of subanesthetic ketamine administration on visual and auditory event-related potentials (ERP) in humans: a systematic review. Front. Behav. Neurosci. 12, 70 (2018).

81. Belujon, P. \& Grace, A. A. Restoring mood balance in depression: ketamine reverses deficit in dopamine-dependent synaptic plasticity. Biol. Psychiatry 76, 927-936 (2014).

82. Brachman, R. A. et al. Ketamine as a prophylactic against stress-induced depressive-like behavior. Biol. Psychiatry 79, 776-786 (2016). 